\title{
In Vitro Analyses of Novel HCN4 Gene Mutations
}

\author{
Melina Möller Nicole Silbernagel $^{\mathrm{b}} \quad$ Eva Wrobel $^{\mathrm{a}} \quad$ Birgit Stallmayer $^{\mathrm{c}}$ \\ Elsie Amedonu ${ }^{a, d}$ Susanne Rinnéb Stefan Peischard ${ }^{a} \quad$ Sven G. Meuth ${ }^{d}$ \\ Bernhard Wünsche Nathalie Strutz-Seebohm ${ }^{\mathrm{a}}$ Niels Decher ${ }^{\mathrm{b}}$ \\ Eric Schulze-Bahrc Guiscard Seebohma
}

aMyocellular Electrophysiology and Molecular Biology, Institute for Genetics of Heart Diseases (IfGH), Department of Cardiovascular Medicine, University Hospital Muenster, Muenster, 'Institute of Physiology and Pathophysiology, Vegetative Physiology Group, Philipps University of Marburg, Marburg, Institute for Genetics of Heart Diseases (IfGH), Department of Cardiovascular Medicine, University Hospital Muenster, Muenster, dDepartment of Neurology, University Hospital Muenster, Muenster, eInstitute of Pharmaceutical and Medical Chemistry, University of Muenster, Muenster, Germany

\section{Key Words}

Channelopathy • Funny current $\left(\mathrm{I}_{\mathrm{f}}\right) \cdot$ Heart - Rhythm - Disease • Bradycardia Arrhythmias • Pacemaking $\cdot \mathrm{HCN}$ Channels

\begin{abstract}
Background/Aims: The hyperpolarization-activated cyclic nucleotide-gated cation channel HCN4 contributes significantly to the generation of basic cardiac electrical activity in the sinus node and is a mediator of modulation by $\beta$-adrenergic stimulation. Heterologous expression of sick sinus syndrome (SSS) and bradycardia associated mutations within the human HCN4 gene results in altered channel function. The main aim was to describe the functional characterization of three (two novel and one known) missense mutations of HCN4 identified in families with SSS. Methods: Here, the two-electrode voltage clamp technique on Xenopus laevis oocytes and confocal imaging on transfected COS7 cells respectively, were used to analyze the functional effects of three $H C N 4$ mutations; $R 378 C, R 550 H$, and E1193Q. Membrane surface expressions of wild type and the mutant channels were assessed by confocal microscopy, chemiluminescence assay, and Western blot in COS7 and HeLa cells. Results: The homomeric mutant channels $R 550 H$ and $E 1193 Q$ showed loss of function through increased rates of deactivation and distinctly reduced surface expression in all three homomeric mutant channels. HCN4 channels containing R55OH and $E 1193 \mathrm{Q}$ mutant subunits only showed minor effects on the voltage dependence and rates of activation/deactivation. In contrast, homomeric R378C exerted a left-shifted activation curve and slowed activation kinetics. These effects were reduced in heteromeric co-expression of $R 378 \mathrm{C}$ with wild-type




\section{Cellular Physiology Cell Physiol Biochem 2018;49:1238-1248 \begin{tabular}{ll} 
and Biochemistry & $\begin{array}{l}\text { DOI: 10.1159/000493301 } \\
\text { Published online: } 6 \text { September, } 2018 \text { www.karger.com/cpb }\end{array}$ \\
\cline { 2 - 3 }
\end{tabular} \\ Möller et al.: Novel HCN4 Mutations}

(WT) channels. Conclusion: Dysfunction of homomeric/heteromeric mutant HCN4-R378C, $R 550 H$, and $E 1193 Q$ channels in the present study was primarily caused by loss of function due to decreased channel surface expression.

\section{Introduction}

The heart beats with astonishing precision throughout human life. During this period, it is adapted to stress situations that increase the rate (positive chronotropic), and pump load (positive inotropic). The sino-atrial node (SAN) is a primary controller of cardiac rhythmicity. It initially develops from a portion of the pro-pacemaking embryonic venous pole under the influence of early canonical Wnt pathway. The adult node is a mixture of distinct cardiomyocytes located at the orifice of the right superior vena cava (SVC) into the right atrium [1]. The cells of the SAN do not reach stable resting membrane potential but show rhythmic activity generated in part by the depolarizing hyperpolarization activated current $I_{\mathrm{f}}\left(\mathrm{I}_{\mathrm{f}}\right.$ - funny current, also named $I_{\mathrm{h}}$ - hyperpolarization activated current or $I_{\mathrm{q}}$ queer current) [2]. This current is generated by the hyperpolarization-activated cyclic nucleotidegated channels of the HCN family. There are four members of this channel family, HCN1-4 which can assemble in native tissues as homo- or hetero-tetramers. The most prominent HCN channel in the human sinus node is HCN4 which may be associated with KCNE2 (MiRP1 - MinK-related peptide-1), a putative $\beta$-subunit of HCN4, in the SAN [1, 2]. Cyclic AMP (cAMP) is generated in response to stress-associated $\beta$-adrenergic stimulation, which then binds to the cyclic nucleotide binding site of HCN4 channels. The binding of cAMP induces conformational HCN4 changes leading to a rightward shift in the steady-state activation curve [3]. The channel becomes more sensitive to hyperpolarizing voltages resulting in the depolarization of SAN cells and faster cardiac rhythm. Impaired pacemaking of the SAN is referred to as sick sinus syndrome (SSS) [3]. SSS is clinically characterized by bradycardia and a combination of symptoms such as fatigue, dizziness, and syncope. It is an inability of the SAN to transmit its activity to the atrium or to generate the intrinsic electrical automaticity [4]. In 2003, familial SSS was linked to inherited mutations in the $H C N$ gene family [5]. Later, the HCN4 locus was identified as a modulator of heart rate in a genome-wide association study (GWAS) [6]. Down-regulation of HCN4 and the subsequent reduction in heart rate has been reported as the mechanism of lowering the resting heart rate associated with endurance exercise [7]. Transgenic mice deficient in HCN1 [8], and HCN2 [9] manifest SSS. In humans, mutations modifying $I_{\mathrm{f}}$ functions are exclusively limited to HCN4 and rarely to KCNE2 [5, 10-20]. Recently, epilepsy-related premature mortality and restrictive cardiomyopathy have been genetically associated with HCN4 mutations [21, 22].

Electrophysiological experiments on heterologously expressed WT and mutant HCN4 channels showed changes in protein trafficking, channel gating, and/or cAMP-sensitivity as the disease mechanism [21]. Here, we described the functional characterization of three (two novel and one known) missense mutations of HCN4 identified in families with SSS.

\section{Materials and Methods}

\section{Mutation identification}

Genomic DNA was extracted from the peripheral lymphocytes of the patients and family members using standard techniques and the HCN4 gene was sequenced as previously described [5].

\section{Molecular biology}

These procedures were as previously described [22]. Human HCN4 was subcloned into the Xenopus laevis Stage V oocyte expression vector pSGEM. The clones were mutated at positions $R 378 C, R 550 H$, and E1193Q by site-directed mutagenesis using QuikChange Lightning Site-Directed Mutagenesis Kit (Agilent Technologies). All constructs were confirmed by automated DNA sequencing. The constructs were 


\section{Cellular Physiology Cell Physiol Biochem 2018;49:1238-1248 \begin{tabular}{l|l|l} 
and Biochemistry Published onlıne: 6 September, 2018 & $\begin{array}{l}\text { (c) } 2018 \text { The Author(s). Published by S. Karger AG, Basel } \\
\text { ww.karger.com/cpb }\end{array}$
\end{tabular} \\ Möller et al.: Novel HCN4 Mutations}

linearized by NheI digest and cRNA was synthesized from $1 \mu \mathrm{g}$ linearized DNA using an in vitro transcription kit (mMessage mMachine T7 kit - Ambion). Concentrations of cRNA were examined by photospectrometry (NanoDrop ND-100), and the quality of the transcript was verified by agarose gel electrophoresis.

\section{Heterologous HCN4 expression in oocytes}

Xenopus laevis Stage V oocytes were obtained from EcoCyte Bioscience, Germany, and injected with $\sim 50 \mathrm{nl}$ of cRNA. The oocytes were incubated at $17^{\circ} \mathrm{C}$ for $3-4$ days. Oocytes were injected with $6 \mathrm{ng}$ HCN4 cRNAs and stored at $(17-18)^{\circ} \mathrm{C}$ in modified ND96-storage solution (in mM: $96 \mathrm{NaCl}, 4 \mathrm{KCl}, 1.8 \mathrm{MgC1}, 0.1$ $\mathrm{CaC1}_{2}, 5$ HEPES (N-[2-hydroxyethyl] piperazine-N'-[2-ethanesulfonic acid]), gentamycin (50 mg/l); $\mathrm{pH}$ 7.6).

\section{Cellular electrophysiology}

Whole-cell currents in Xenopus oocytes were recorded with standard two-electrode-voltageclamp techniques using the Turbo TEC10-CX amplifier (NPI) at room temperature $(22-24)^{\circ} \mathrm{C}$. Data was acquired with Clampex (pCLAMP 8.0, Axon Instruments), and analyzed with ClampFit (pCLAMP 8.0, Axon Instruments), Prism 6.1 software (GraphPad Software, San Diego, California, USA), and Origin 8.0 (Microcal Inc.). Whole-cell currents were recorded in ND96-recording solution (in mM: $96 \mathrm{NaCl}, 4 \mathrm{KCl}, 1.8 \mathrm{MgC} 1_{2}, 0.1$ $\mathrm{CaC1}_{2}, 5$ HEPES; $\mathrm{pH}$ 7.6).

Peak tail current analysis at a potential of $-140 \mathrm{mV}$ after varying test pulses was used to assess the voltage dependence of the HCN4 channel activation. These peak currents were fitted to the standard Boltzmann equation to calculate the voltage dependence of activation. $I_{t}=\left(1-\mathrm{min}-P_{0} /\left[1-\exp \left(\left(\mathrm{V}-\mathrm{V}_{1 / 2}\right) / \mathrm{k}\right)\right]\right.$ $\min -P_{0}$, to obtain the voltage required for half-maximal activation $\left(\mathrm{V}_{1 / 2}\right)$, slope factor $(k)$, and the minimum open probability (min-Po, defined as the minimum value of relative tail current [23].

HCN4 channel activation and deactivation was analyzed by fitting activating inward currents or the upward reflection upon deactivating pulses to an exponential equation of the form: $I(t)=a \exp (-t / \tau)+a_{0}$, with a fast time constant, $\tau$; their respective amplitudes, $a$; and an offset current, $a_{0}$.

\section{Confocal microscopy, chemiluminescence assay, and Western blot in mammalian cells}

Cells were cultivated in petri dishes at $37^{\circ} \mathrm{C}$ and $5 \% \mathrm{CO}_{2}$ in DMEM medium $+10 \%$ FBS and $1 \%$ MEM Non-essential amino acid (Sigma-Aldrich). During culturing, cells were carefully washed in $10 \mathrm{ml}$ PBS (Sigma-Aldrich) prior to the addition of new culture medium. COS7 cells were then transfected with the HA-EGFP-HCN4 construct (1 $\mu$ g HCN4 cDNA), [24] and in the case of confocal microscopic experiments, additional $0.5 \mu \mathrm{g}$ ER-Marker cDNA using jetPRIME (Peqlab) was transfected. Chemiluminescence assay: HeLa cells were transfected with the HA-EGFP-HCN4 construct (1 $\mu \mathrm{g}$ HCN4 cDNA). This assay was performed as previously described [25]: After $48 \mathrm{~h}$, HeLa cells were fixed with 4\% PFA (in PBS), washed three times with PBS, and blocked with $10 \%$ normal goat serum (in PBS supplemented with $0.1 \%$ sodium azide). Cells were stained with a monoclonal anti-HA primary antibody (HA probe (F-7), Santa Cruz Biotechnology) and washed intensely with PBS. As a secondary antibody, an HRP-conjugated antibody (goat anti-mouse IgGHRP, Santa Cruz Biotechnology) was used. After several washing steps with PBS, surface expression was measured in a luminometer (Glomax 20/20, Promega) using a luminogenic substrate (SuperSignal ELISA Femto, Pierce Biotechnology). Western blot analysis: Protein lysates were separated on a SDS-polyacrylamide gel and transferred to a nitrocellulose membrane via a Semi-Dry Transfer Cell (Biorad). HA-EGFP-HCN4 protein was visualized by immunoblotting with mouse anti-GFP antibody (ab290, 1:5000, Abcam). The binding of the primary antibody was detected using peroxidase-conjugated goat anti-rabbit IgG antibody (\#32460, 1:2000, Pierce Thermo Scientific) and a chemiluminescent extended-duration substrate (Super Signal West Dura, Thermo Scientific).

Confocal microscopy: COS7 cells were fixed with 4\% PFA (in PBS), washed three times with PBS, and blocked with $10 \%$ normal goat serum (in PBS supplemented with $0.1 \%$ sodium azide). Cells were incubated for $1 \mathrm{~h}$ in monoclonal anti-HA primary antibody (HA probe (F-7), Santa Cruz Biotechnology), washed three times in PBS and stained with secondary dye-conjugated antibody (Alexa Fluor® 594 Dye, 1:200) in 5\% goat serum in PBS. Prior to fixation, the cells were washed three times in PBS and embedded in Fluoromount ${ }^{\mathrm{TM}}$ (Sigma-Aldrich). Confocal microscopy was performed using a DMI4000B (Leica) microscope with a 40x objective (HCX PL FLUOTAR 40.0×0.75), excitation was at $488 \mathrm{~nm}$, and fluorescence was detected between 550 and $594 \mathrm{~nm}$. Data was analyzed using ImageJ software (U.S. National Institutes of Health, Bethesda, MD, USA). Line scans were used to analyze the total HCN4. 


\section{Cellular Physiology Cell Physiol Biochem 2018;49:1238-1248 \begin{tabular}{l|l|l} 
and Biochemistry Published onlıne: 6 September, 2018 & $\begin{array}{l}\text { (c) } 2018 \text { The Author(s). Published by S. Karger AG, Basel } \\
\text { ww.karger.com/cpb }\end{array}$
\end{tabular}

\section{Molecular modeling}

A consensus homology HCN4 model was generated. Several individual modeling steps using YASARA Structure version 10.1 were required. The HCN4 pore domain was generated as a consensus model using a modelling protocol as previously described [26]. This pore domain was fused to a HCN4-C-terminal consensus model using the same protocol. The resultant model represented one human HCN4-subunit covering residues 249 - 734. A tetrameric channel HCN4 model was generated by combining four virtual identical subunits in a 4-fold symmetry. The resulting tetrameric model was energy minimized using the YASARA Structure Module Energy Minimization Server. By swapping residues R378 to cys or R550 to his, mutant channels were generated. WT and mutant channel models were incorporated into membranes and short molecular dynamics (MD) simulations (5 ns) were performed as previously described [27]. Only local structural changes were noted at the end of the simulations when comparing the WT channels structure with those of the mutant channels.

\section{Statistical analyses}

These were performed using unpaired t-test and one-way-ANOVA. Data was presented as mean values, and error bars indicated SEM. Significance levels of the test showing $p$-values were indicated by stars as follows: ${ }^{*}=p<0.05,{ }^{* *}=p<0.01,{ }^{* * *}=p<0.001,{ }^{* * * *}=p<0.0001$.

\section{Results}

\section{Identification of the three HCN4} gene mutations in patients

Over the years, patients with clinical manifestations of SSS were identified through the outpatient service; they all exhibited substantially reduced heart rate lower than 60 beats per minute at rest after the exclusion of secondary causes. The classical candidate gene for SSS is the HCN4 gene [5]. In summary, a genetic analysis of the complete $H C N 4$ gene in 57 unrelated probands with idiopathic or familial SSS was performed, among them, three nucleotide variants resulting in non-synonymous amino acid exchanges were found. Two (HCN4R378C, and HCN4-R550H) were novel, whereas HCN4-E1193Q had been recently published in a proband with early-onset atrial fibrillation, Brugada syndrome, with epilepsy, and with restrictive cardiomyopathy [12]. The mutations were positioned in the transmembrane domain (HCN4-R378C) or in the intracellular C-terminus (HCN4-R55OH and HCN4-E1193Q), (Fig. 1A).

In a female proband with childhood onset of SSS, the heterozygous nucleotide

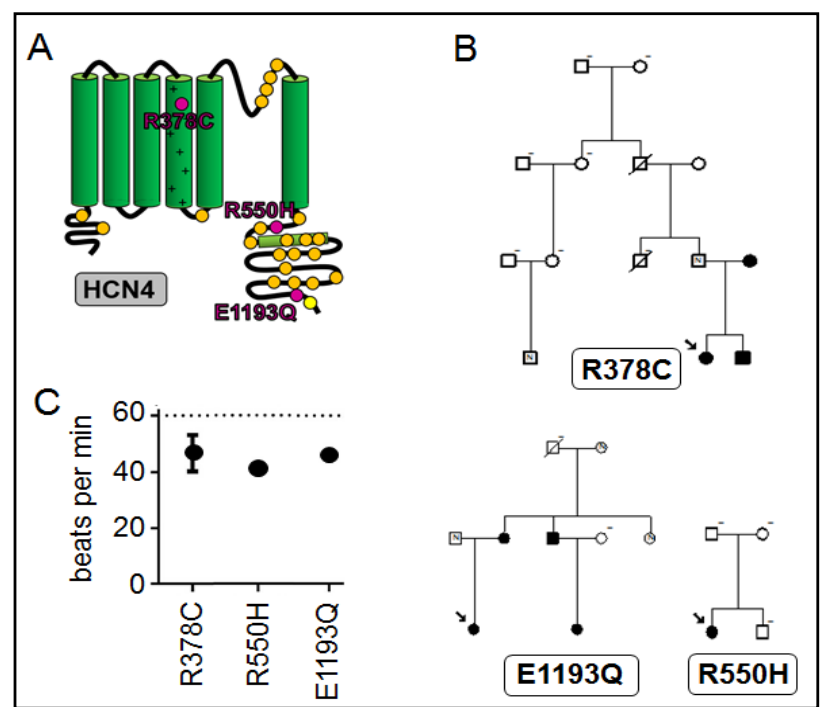

Fig. 1. Localization of HCN4 gene mutations. In the three SSSfamilies, mutations in HCN4 were identified. A. Localization of published gene variants [23] and the gene variants of interest are marked as yellow and magenta, respectively in the cartoon on HCN4 subunit. B. The pedigrees of families with their respectively identified gene variant is shown. Males are represented by squares, females by circles. Mutation carriers are indicated by solid symbols, $\mathrm{N}$ symbol indicates absence of HCN4 mutation (wild type). "-" at the right indicates family member who refused genetic testing. Scratched symbols indicate deceased family members. Arrow indicates index patient. All three genetically verified R378C patients were diagnosed with sinus bradycardia. $\mathrm{C}$. The number of heart beats per minute (resting heart rate) was analyzed and presented as mean values +/- SEM from the index patients (black arrows). 


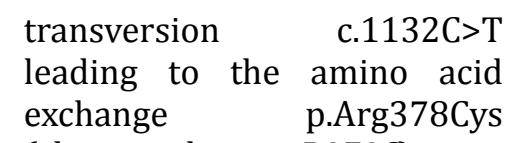
(shortened as: R378C) was identified. The patient received a pacemaker at the age of 18 for recurrent syncope due to symptomatic SSS. The nucleotide exchange in an autosomal-dominant pattern co-segregated with the disease in the family and was detected in the proband's brother and mother. R378C (FID 11082) is extremely rare in the Exome Aggregation Consortium (ExAC) database (minor allele frequency - MAF: 0.0025\%) and concordantly predicted to be "deleterious" by several mutation pathogenicity tools (SIFT, Mutation taster, and PolyPhen-2).

The novel nonsynonymous amino acid exchange p.Arg550His (shortened as: R550H) (FID 11079) (c.1649G $>$ A) was heterozygously identified in a female proband with asymptomatic sinus bradycardia and ventricular extrasystoles (VES). Its characteristics include extreme rarity in the ExAC database and "deleterious" prediction by the mutation pathogenicity tools, as well as high degree of evolutionary conservation of arginine at position 550 throughout the HCN4 orthologs.

Finally, we identified a heterozygous nucleotide transversion c.3577G>C (resulting in the non-synonymous amino acid exchange p.Glu1193Gln, shortened as: E1193Q) in a 22-year-old female proband with symptomatic SSS. Her clinical symptoms included recurrent syncope with first manifestation at age of 14 . The mutation was also identified in three other family members (FID 11034). The low-frequency variant, E1193Q was recently described as a pathogenic mutation in a patient with early-onset atrial fibrillation, but it exists in control population databases such as the Exome Variant Server (MAF: 0.02\%) or ExAC (MAF: 0.099\%), raising its functional impact.

Since all three HCN4 variants had not been reported before in the context of SSS, we analyzed their functional effects to further support the potential disease-causing mutations notion.

\section{Novel HCN4 mutations reduced function in heterologous expression systems}

To assess the functional consequences of the HCN4 mutations, we analyzed the homoand hetero-meric mutant channels in heterologous expression systems. Homomeric expression of native HCN4 (HCN4-WT) in Xenopus laevis oocytes resulted in classical hyperpolarization-activated channel currents (Fig. 2). In all the cases, expression of mutant HCN4 channels resulted in decreased, but fully-activated currents compared to HCN4-WT. Specifically, R378C reduced HCN4-mediated currents by 70\% (homomeric state), and 57\% (heteromeric), $\mathrm{R} 550 \mathrm{H}$ reduced currents by 49\% (homomeric), and 29\% (heteromeric), while E1193Q reduced currents by $52 \%$ (homomeric) and $49 \%$ (heteromeric), respectively. 


\section{Cellular Physiology Cell Physiol Biochem 2018;49:1238-1248 \begin{tabular}{ll|l} 
and Biochemistry Published onlIne: 6 September, 2018 & (c) 2018 The Author(s). Published by S. Karger AG, Basel \\
ww.karger.com/cpb
\end{tabular}

Novel HCN4 mutations altered biophysical behaviour of mutant channels in heterologous expression systems

It has been reported that some human HCN4 mutations cause altered HCN4 channel biophysical behavior [21]. We analyzed voltage-dependence and found an extensive significant left-shifted $\mathrm{V}_{1 / 2}$ for the $R 378 \mathrm{C}$ mutant $\left(\mathrm{V}_{1 / 2}\right.$-shift $=-32.5^{1 / 2} \mathrm{mV}, \mathrm{R} 378 \mathrm{C} / \mathrm{WT}$ : $\mathrm{V}_{1 / 2}$-shift $=-7.9 \mathrm{mV}$, Fig. 3). Time constants of activation $\tau_{\text {act }}$ were increased in R378C and deactivation $\tau_{\text {deact }}$ were decreased in most homo- and hetero-meric mutant channels compared to those of WT (Fig. 4). Notably, the slowing of the mutant channel activation in case of $E 1193 Q$ only occurred outside the physiological range of membrane potentials.

Novel HCN4 mutations reduced channel plasma membrane expression in heterologous expression systems

Reduced channel surface expression is often seen in diseases associated with ion channels. Here, we used HCN4 clones with an external HA-tag and an internal EGFP as previously described [24]. WT and mutant channels were expressed in HeLa cells using the external HA-tag to detect the proteins at the plasma membrane by a chemiluminescence assay. Plasma membrane abundance of all three mutant channels was significantly diminished (Fig. 5A). Western blot analyses of the cells detecting the $\mathrm{N}$-terminal EGFP-tag showed decreased protein expression, indicating that the mutant HCN4 channels had impaired protein stability due to their respective mutations (Fig. 5A). This effect was particularly evident for the HCN4-E1193Q mutant. Reduced plasma membrane expression of mutant $H C N 4$ channels possibly resulted from altered subcellular localization, in addition to the reduced total HCN4 protein expression. Thus, to test the altered subcellular localization, WT and mutant channels were expressed in COS7 cells and the external HA-tag was used for immunostaining. Line plots of the EGFP and red antibody fluorescence generated information on plasma membrane expressed HCN4 channels as well as those throughout the cell. The ratio between the peak membrane EGFP intensity and the mean EGFP intensity was calculated to estimate the relative membrane expression of WT and mutant channels. The resulting relative plasma membrane abundance of mutant HCN4 channels were reduced similar to those of the biotinylation Western blot results (Fig. 5). Inverted ratios (increased channel protein intracellular vs. channel protein at the plasma membrane) were found with HCN4-R378C and HCN4-E1193Q, suggesting trafficking defects in mutant HCN4 channels. Trafficking defects of ion channels are often associated with time-dependent channel endocytosis from the cell membrane. We, therefore expressed WT and mutant HCN4 channels in Xenopus laevis oocytes and recorded current amplitudes 3 - 6 days after cRNA injections. Whereas functional expression of HCN4-WT increased during this period, mutant channels tend to showed decreased function on day 6 . This effect seemed to be more prominent in HCN4-R378C and HCN4-R550H than in HCN4-E1193Q, suggesting reduced protein stability (Fig. 6).

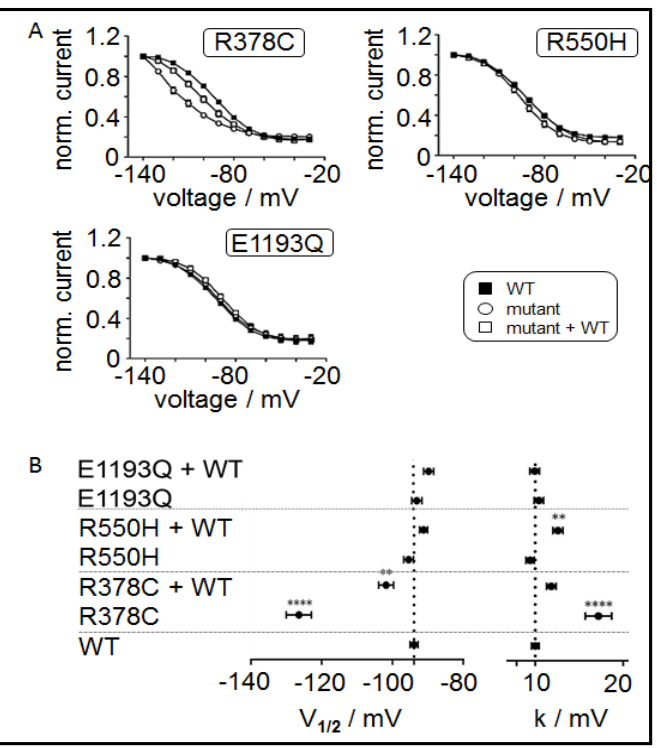

Fig. 3. Voltage dependence of HCN4-WT and mutant HCN4 channels in Xenopus laevis oocytes. normalized and plotted against the test pulse potential. B. The initial tail currents of individual cells were determined and fitted to a Boltzmann function. The individual values were used to calculate the mean $\mathrm{V}_{1 / 2}, \mathrm{k}$ values, and SEM as well as perform statistical tests. The mean values of WT are indicated by dotted lines. Data was presented as mean +/- SEM $(n=18-45)$. 
Fig. 4. Time constants of activation and deactivation of wild type and mutant HCN4 channels. Time constant of activation during $3 \mathrm{~s}$ hyperpolarizing pulses at different voltages and time constant of deactivation after $3 \mathrm{~s}$ activating pulses $(-140 \mathrm{mV})$ were determined by fit single-exponential models. Time constants at indicated voltage are presented as bar diagrams and asterisks indicate significant changes with respect to wild type. Data was presented as mean +/- SEM $\left(\tau_{\text {act }}: \mathrm{n}=11-17 ; \tau_{\text {deact }}: \mathrm{n}=8-11\right)$.

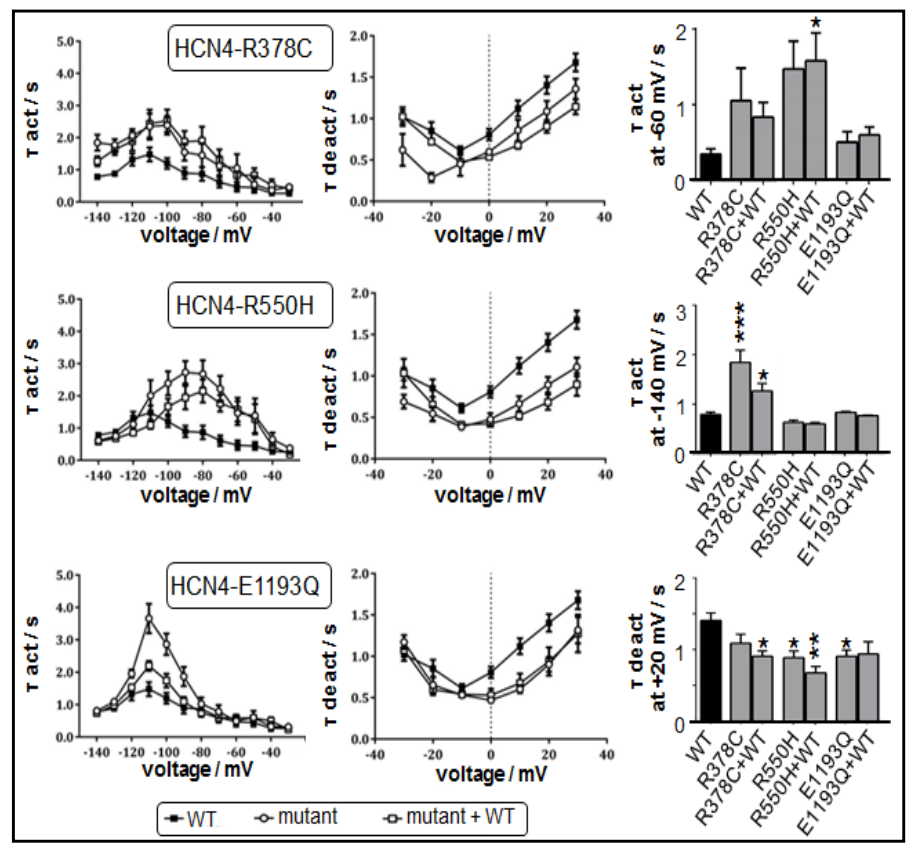

Fig. 5. HCN4 membrane expression determined by Western blot, chemiluminescence assay, and confocal imaging. A. HCN4 surface and total protein expression was assayed using biotinylation and Western blot analysis. The representative Western blot is shown on the left. The right panel shows the mean relative light units (RLUs) of 4 consecutive luminescence assays for the determination of surface expression. Double and triple asterisks in Fig. 5A indicate a statistically significant decrease with respect to WT. B. Confocal imaging was used to detect the fluorescence (red) of the antibody and EGFP. Line scans using the anti-HA signal allowed for identification of the plasma membrane fraction of the HCN4 channels.

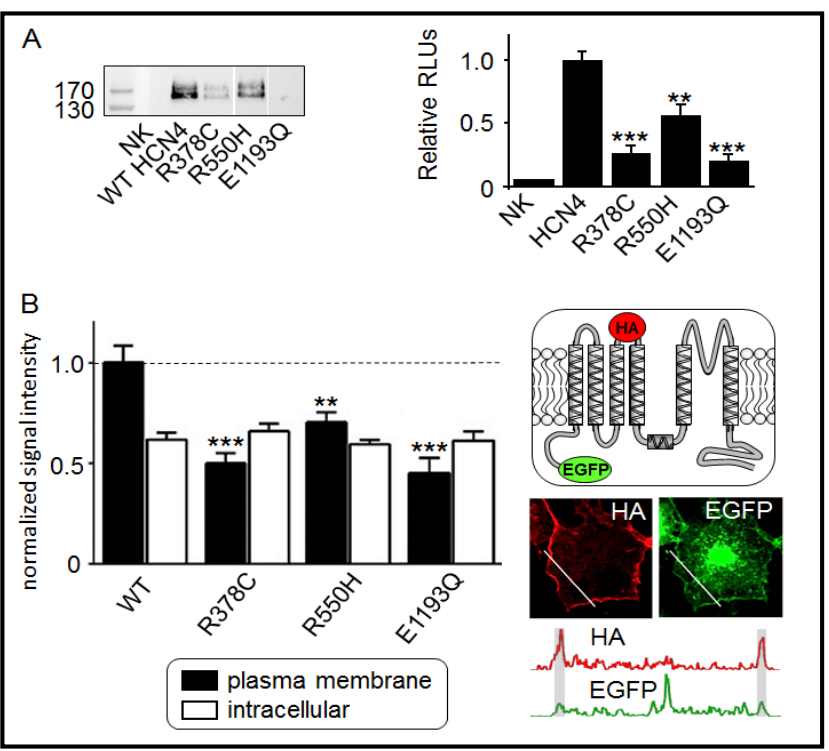

The EGFP intensity at this position was

defined as plasma membrane-incorporated HCN4. The mean EGFP-signal intensity between the plasma membrane intensity peaks was defined as intracellular (cytosolic) HCN4 fraction. The relative intensities normalized to WT-cytoplasmic membrane signals are shown in the left panel. All cells were treated in parallel using identical conditions (transfections, antibody application, microscope settings etc.). Data was presented as mean + /- SEM, $n=68-75$ ). Results of statistical comparison of plasma membrane signal and the intracellular signal of mutants with the respective WT was performed and results are indicated by asterisks.

The novel HCN4 mutations possibly altered structural features of mutant channels as suggested by in silico modelling

The mutations reported here were localized in different regions spanning from the transmembrane domain to the end of the C-terminus (Fig. 1A). Kinetic phenotypes were relatively variable as well. However, they all showed physiologically reduced plasma membrane expression. To understand the structural basis of channel dysfunction and 
altered localizations, homology modeling was performed, followed by molecular dynamics (MD) simulations on WT and mutant channels which were run in parallel and the dynamic characteristics compared on a small-time scale. High-resolution consensus homology modeling was possible for residues 249 - 734. Therefore, only altered residues $R 378 \mathrm{C}$ and $R 550 H$ could be modelled. We found that $R 378 \mathrm{C}$ and $R 550 \mathrm{H}$ were localized to the center of the voltage sensor and gating ring domains, respectively (Fig. 7). R378C may have contact with the outer pore domain of the adjacent subunit (within 5Å- LEU 438: 92 contacts, GLN 443 : 83 contacts, ASP 444 : 46 contacts, VAL 439 : 40 contacts, PHE 445 : 1 contact), whereas $R 550 H$ was in contact with the cAMP binding domain within one subunit (within 5Å-GLN 617 : 128 contacts, PRO 618 : 29 contacts, ASP 620 : 28 contacts, GLY $619: 14$ contacts, TYR $678: 1$ contact). In silico simulation suggests that the $R 378 C$ and $R 550 H$ contacts may have been affected by the diseaseassociated mutations. However, the precise changes were local and did not affect the rest of the modelled protein structure. This may have been due to the relatively short simulation duration, as only extremely cost intensive long-range simulations within microseconds could uncover global changes. In summary, mutant HCN4 channels showed reduced function in the heterologous expression systems. Besides variable kinetic effects, all mutant channels exerted reduced cell surface expressions which may be the main reason for the loss of function in the mutant channels leading to SSS.

\section{Discussion}

Bradycardia is a major cause of cardiac arrhythmias and sudden cardiac death. Mostly, it results from the reduced rhythmic activity of the sinus node rather than as a developmental defect. Determinants of spontaneous diastolic depolarization and rhythmic (pacemaking) activity of the human sinus node are the hyperpolarization-activated current $I_{\mathrm{f}}$ (mediated

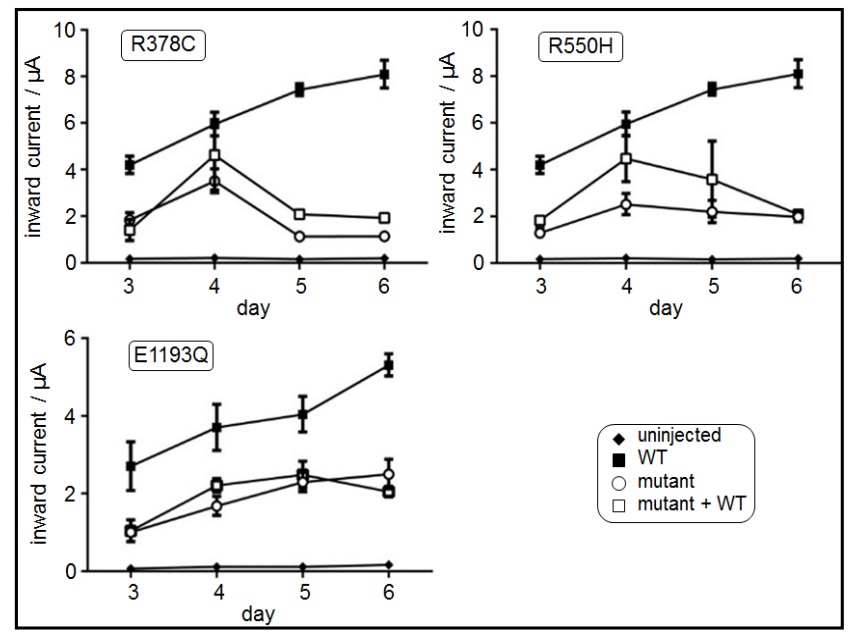

Fig. 6. Current amplitudes at $-140 \mathrm{mV}$ of HCN4-WT and mutant HCN4 channels measured for four subsequent days. Oocytes were injected with WT and mutant HCN4 channel cRNA and function of channels were assayed 3 - 6 days after the injections. Data was presented as mean +/- SEM, all values $\mathrm{n}=9$.

Fig. 7. 3D-simulated HCN4 channels and analysis. Consensus homology models of the voltage sensor, pore, and intracellular domains were generated and fused covering residues 249-734. The resultant model was energy minimized. Shown below are the positions of two of the identified residues ( $R 378, \mathrm{R} 550)$ in the voltage sensor domain and gating ring, respectively. The backbone of one HCN4 subunit is depicted in ribbon representation. CAMP is shown in volumefill and CPK coloring.

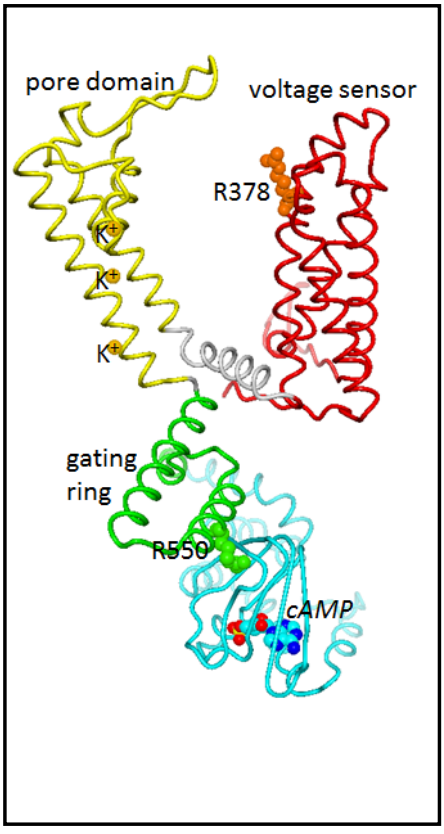




\section{Cellular Physiology Cell Physiol Biochem 2018;49:1238-1248

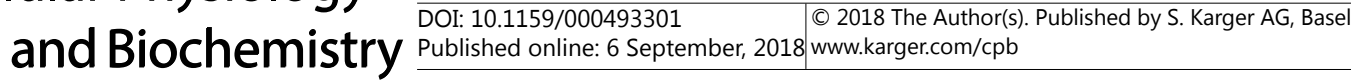 \\ Möller et al.: Novel HCN4 Mutations}

by HCN 4 channels) and the inward calcium currents. Consequently, reduced HCN4 function results in decreased rate of sinus node activity. Reduced HCN4 function has been reported for several familial HCN4 mutations associated with bradycardia in SSS [21]. Furthermore, reduced $I_{\mathrm{f}}$ function in the human sinus node action potentials leads to bradycardia which can be calculated in silico using a recently developed sinus node model [28]. Consistently, we three rare $H C N 4$ gene variants in four SSS patients. These gene variants were clearly inherited in the cases of HCN4-R378C and HCN4-E1193Q as the gene trees showed normal inheritance with 3 - 4 family members carrying the variant (Fig. 1B). Due to lack of clinical data in case of the E1193Q mutation and the R550H mutation, it is unclear whether the genetically affected family members also show sinus bradycardia.

As $H C N 4$ gene mutations potentially cause reduced $I_{\mathrm{f}}$ function and sinus node rhythmicity, in vitro experiments were used to assay the identified gene variants. Significantly reduced fully-activated current in all three gene variants were found (Fig. 2). Kinetic analysis of activation showed greater left-shifted activation for homomeric $R 378 \mathrm{C}$ and to less extent for heteromeric R378C/WT complexes (Fig. 3). Residue 378 is positioned in the S4 transmembrane segment (the primary voltage sensor), thus, the shift in voltage dependence was conceivable. Positively-charged arginines in the S4 segment of HCN channels allow for the inverted opening of HCN channels compared to those of the prototypic Kv [23]. Thus, the positively-charged $R 378 \mathrm{C}$ mutated in the patient increased the apparent voltage sensation by charge neutralization effect. Activation of mutants R378C and R550H tend to be slowed down at $-60 \mathrm{mV}$, while deactivation at $20 \mathrm{mV}$ of all three mutants show a trend to get accelerated (Fig. 4). Data was consistent with destabilization of the channels open state, an effect that shifts the fraction of channels towards the closed state resulting in reduced number of active channels at the plasma membrane.

Aside from kinetic effects, channel function was determined by the channel abundance at the plasma membrane. All three mutations led to reduced plasma membrane abundance as assayed by Western blot, chemiluminescence, and confocal imaging (Fig. 5). In WT channels, protein density at the plasma membrane was increased compared to that of intracellular protein. In mutant channels, the intracellular protein levels were comparable to that of WT. However, in general less plasma membrane protein was observed in mutant channels compared to WT (Fig. 5). Furthermore, time-dependent reduction of mutant HCN4 channel currents suggests altered trafficking, and possibly increased endocytosis (Fig. 6). These results show that altered trafficking e.g. decreased exocytosis or increased endocytosis of mutant HCN4 leads to decreased channel surface expression and reduced channel function in vitro. E1193Q mutation in association with Brugada syndrome has also been reported [29]. Macri et al. found an association between HCN4-E1193Q mutation and atrial fibrillation and patch clamp experiments were used to assess the mutation-induced changes in channel properties [12]. Their study found a mild but insignificant shift in the $\mathrm{V}_{1 / 2}$ which is consistent with our results. Further, HCN4-E1193Q mutation was associated with epilepsy and restrictive cardiomyopathy $[21,22]$. The loss of function of the in vitro experimental data on E1193Q mutation presented here are in line with the findings by Bagnall et al. (2016) and Tarnovskaya et al. (2017) [21, 22]. In the present study, there was no association between this mutation and Brugada syndrome, atrial fibrillation, epilepsy or restrictive cardiomyopathy. Possibly, the HCN4-E1193Q mutation is a genetic modifier of the other clinical entities reported earlier as well as a mutation that causes clear functional alterations in HCN4 [21, 22].

Homology modeling of HCN4 channels showed that R378 interacts with the pore domain of the adjacent HCN4 subunit. R550 within the gating ring is in contact with the cAMP binding domain. Thus, both residues are present at the interface of the two channel domains in an in-silico model and mutations at these sites alter inter-domain assembly. Probably, such altered domain-domain interactions cause protein misfolding which decrease protein stability within the cellular context.

Here, we identified the mild kinetic effects and the markedly reduced plasma membrane abundance as the major causes of the mutant HCN4 channel dysfunction. The moderate 


\section{Cellular Physiology Cell Physiol Biochem 2018;49:1238-1248 $\begin{array}{ll}\text { and Biochemistry } & \text { DOI 10.1159/000493301 } \\ \text { Published online: } 6 \text { S September, } 2018 \text { www.karger.com/cpb }\end{array}$ \\ Möller et al.: Novel HCN4 Mutations}

hyperpolarizing shift in half-activation voltage of $R 378 C+W T$ (shift compared to WT $-7.9 \pm$ $2.1 \mathrm{mV}$ ) is slightly larger compared to the less prominent shift in HCN4-573X and HCN4$695 X$ reported with SSS $[5,17]$. However, the loss of function in HCN4-R378C resulted from the reduced surface expression rather than impaired cAMP modulation, which caused functional impairments in HCN4-573X or HCN4-695X. Notably, none of the three mutations analyzed here had dramatic impairments in cAMP activation as the injection of cAMP led to inestimable degree activation of WT and mutant currents, (data not shown). HCN4- $R 550 \mathrm{H}$ is positioned in close proximity to the reported HCN4-D553N mutation. As opposed to HCN4$R 550 H, D 553 N$ caused loss of function mainly due to the defective C-linker mediated gating ring and trafficking [24].

In summary, we analyzed three HCN4 mutations identified in index patients that caused loss of function in vitro. Loss of function may have resulted from significantly reduced functional HCN4 channel availability and cell surface expression due to defective trafficking.

\section{Acknowledgements}

We acknowledge the grant support through IMF-SE 111712 and DFG-SE1077-3/3 to GS and DFG, INST 2105/27-1 to SGM.

\section{Disclosure Statement}

No conflict of interests exists.

\section{References}

1 Bressan M, Liu G, Mikawa T: Early mesodermal cues assign avian cardiac pacemaker fate potential in a tertiary heart field. Science 2013;340:744-748.

-2 Morad M, Zhang XH: Mechanisms of spontaneous pacing: Sinoatrial nodal cells, neonatal cardiomyocytes, and human stem cell derived cardiomyocytes. Can J Physiol Pharmacol 2017:1-8.

3 Sartiani L, Romanelli MN, Mugelli A, Cerbai E: Updates on hon channels in the heart: Function, dysfunction and pharmacology. Curr Drug Targets 2015;16:868-876.

4 Bigger JT, Jr., Reiffel JA: Sick sinus syndrome. Annu Rev Med 1979;30:91-118.

5 Schulze-Bahr E, Neu A, Friederich P, Kaupp UB, Breithardt G, Pongs O, Isbrandt D: Pacemaker channel dysfunction in a patient with sinus node disease. J Clin Invest 2003;111:1537-1545.

6 den Hoed M, Eijgelsheim M, Esko T, Brundel BJ, Peal DS, Evans DM, Nolte IM, Segre AV, Holm H, Handsaker RE, Westra HJ, Johnson T, Isaacs A, Yang J, Lundby A, Zhao JH, Kim YJ, Go MJ, Almgren P, Bochud M et al.: Identification of heart rate-associated loci and their effects on cardiac conduction and rhythm disorders. Nat Genet 2013;45:621-631.

7 D'Souza A, Bucchi A, Johnsen AB, Logantha SJ, Monfredi O, Yanni J, Prehar S, Hart G, Cartwright E, Wisloff U, Dobryznski H, DiFrancesco D, Morris GM, Boyett MR: Exercise training reduces resting heart rate via downregulation of the funny channel hcn4. Nat Commun 2014;5:3775.

-8 Fenske S, Krause SC, Hassan SI, Becirovic E, Auer F, Bernard R, Kupatt C, Lange P, Ziegler T, Wotjak CT, Zhang H, Hammelmann V, Paparizos C, Biel M, Wahl-Schott CA: Sick sinus syndrome in hcn1-deficient mice. Circulation 2013;128:2585-2594.

-9 Ludwig A, Budde T, Stieber J, Moosmang S, Wahl C, Holthoff K, Langebartels A, Wotjak C, Munsch T, Zong X, Feil S, Feil R, Lancel M, Chien KR, Konnerth A, Pape HC, Biel M, Hofmann F: Absence epilepsy and sinus dysrhythmia in mice lacking the pacemaker channel hcn2. EMBO J 2003;22:216-224.

10 Duhme N, Schweizer PA, Thomas D, Becker R, Schroter J, Barends TR, Schlichting I, Draguhn A, Bruehl C, Katus HA, Koenen M: Altered hcn4 channel c-linker interaction is associated with familial tachycardiabradycardia syndrome and atrial fibrillation. Eur Heart J 2013;34:2768-2775. 


\section{Cellular Physiology Cell Physiol Biochem 2018;49:1238-1248 \begin{tabular}{ll} 
DOI: 10.1159/000493301 & O 2018 The Author(s). Published by S. Karger AG, Basel \\
and Biochemistry & Published online: 6 September, 2018 www.karger.com/cpb \\
\cline { 2 - 3 }
\end{tabular} \\ Möller et al.: Novel HCN4 Mutations}

11 Laish-Farkash A, Glikson M, Brass D, Marek-Yagel D, Pras E, Dascal N, Antzelevitch C, Nof E, Reznik H, Eldar M, Luria D: A novel mutation in the hcn4 gene causes symptomatic sinus bradycardia in moroccan jews. J Cardiovasc Electrophysiol 2010;21:1365-1372.

$>12$ Macri V, Mahida SN, Zhang ML, Sinner MF, Dolmatova EV, Tucker NR, McLellan M, Shea MA, Milan DJ, Lunetta KL, Benjamin EJ, Ellinor PT: A novel trafficking-defective hcn4 mutation is associated with earlyonset atrial fibrillation. Heart Rhythm 2014;11:1055-1062.

-13 Milanesi R, Baruscotti M, Gnecchi-Ruscone T, DiFrancesco D: Familial sinus bradycardia associated with a mutation in the cardiac pacemaker channel. N Engl J Med 2006;354:151-157.

-14 Milano A, Vermeer AM, Lodder EM, Barc J, Verkerk AO, Postma AV, van der Bilt IA, Baars MJ, van Haelst PL, Caliskan K, Hoedemaekers YM, Le Scouarnec S, Redon R, Pinto YM, Christiaans I, Wilde AA, Bezzina CR: Hcn4 mutations in multiple families with bradycardia and left ventricular noncompaction cardiomyopathy. J Am Coll Cardiol 2014;64:745-756.

15 Nawathe PA, Kryukova Y, Oren RV, Milanesi R, Clancy CE, Lu JT, Moss AJ, Difrancesco D, Robinson RB: An lqts6 mirp1 mutation suppresses pacemaker current and is associated with sinus bradycardia. J Cardiovasc Electrophysiol 2013;24:1021-1027.

16 Nof E, Luria D, Brass D, Marek D, Lahat H, Reznik-Wolf H, Pras E, Dascal N, Eldar M, Glikson M: Point mutation in the hcn 4 cardiac ion channel pore affecting synthesis, trafficking, and functional expression is associated with familial asymptomatic sinus bradycardia. Circulation 2007;116:463-470.

17 Schweizer PA, Duhme N, Thomas D, Becker R, Zehelein J, Draguhn A, Bruehl C, Katus HA, Koenen M: Camp sensitivity of hcn pacemaker channels determines basal heart rate but is not critical for autonomic rate control. Circ Arrhythm Electrophysiol 2010;3:542-552.

18 Schweizer PA, Schroter J, Greiner S, Haas J, Yampolsky P, Mereles D, Buss SJ, Seyler C, Bruehl C, Draguhn A, Koenen M, Meder B, Katus HA, Thomas D: The symptom complex of familial sinus node dysfunction and myocardial noncompaction is associated with mutations in the hen4 channel. J Am Coll Cardiol 2014;64:757-767.

19 Ueda K, Nakamura K, Hayashi T, Inagaki N, Takahashi M, Arimura T, Morita H, Higashiuesato Y, Hirano Y, Yasunami M, Takishita S, Yamashina A, Ohe T, Sunamori M, Hiraoka M, Kimura A: Functional characterization of a trafficking-defective hcn4 mutation, d553n, associated with cardiac arrhythmia. J Biol Chem 2004;279:27194-27198.

20 Zhou J, Ding WG, Makiyama T, Miyamoto A, Matsumoto Y, Kimura H, Tarutani Y, Zhao J, Wu J, Zang WJ, Matsuura H, Horie M: A novel hcn4 mutation, g1097w, is associated with atrioventricular block. Circ J 2014;78:938-942.

21 Verkerk A0, Wilders R: Pacemaker activity of the human sinoatrial node: An update on the effects of mutations in hon4 on the hyperpolarization-activated current. Int J Mol Sci 2015;16:3071-3094.

22 Seebohm G, Scherer CR, Busch AE, Lerche C: Identification of specific pore residues mediating kcnq1 inactivation. A novel mechanism for long qt syndrome. J Biol Chem 2001;276:13600-13605.

-23 Chen J, Piper DR, Sanguinetti MC: Voltage sensing and activation gating of hcn pacemaker channels. Trends Cardiovasc Med 2002;12:42-45.

-24 Netter MF, Zuzarte M, Schlichthorl G, Klocker N, Decher N: The hcn4 channel mutation d553n associated with bradycardia has a c-linker mediated gating defect. Cell Physiol Biochem 2012;30:1227-1240.

-25 Streit AK, Matschke LA, Dolga AM, Rinne S, Decher N: Rna editing in the central cavity as a mechanism to regulate surface expression of the voltage-gated potassium channel kv1.1. J Biol Chem 2014;289:2676226771.

26 Henrion U, Zumhagen S, Steinke K, Strutz-Seebohm N, Stallmeyer B, Lang F, Schulze-Bahr E, Seebohm G: Overlapping cardiac phenotype associated with a familial mutation in the voltage sensor of the kcnq1 channel. Cell Physiol Biochem 2012;29:809-818.

-27 Strutz-Seebohm N, Pusch M, Wolf S, Stoll R, Tapken D, Gerwert K, Attali B, Seebohm G: Structural basis of slow activation gating in the cardiac i ks channel complex. Cell Physiol Biochem 2011;27:443-452.

28 Verkerk AO, Wilders R: Hyperpolarization-activated current, if, in mathematical models of rabbit sinoatrial node pacemaker cells. Biomed Res Int 2013;2013:872454.

29 Allegue C, Coll M, Mates J, Campuzano O, Iglesias A, Sobrino B, Brion M, Amigo J, Carracedo A, Brugada P, Brugada J, Brugada R: Genetic analysis of arrhythmogenic diseases in the era of ngs: The complexity of clinical decision-making in brugada syndrome. PloS one 2015;10:e0133037. 